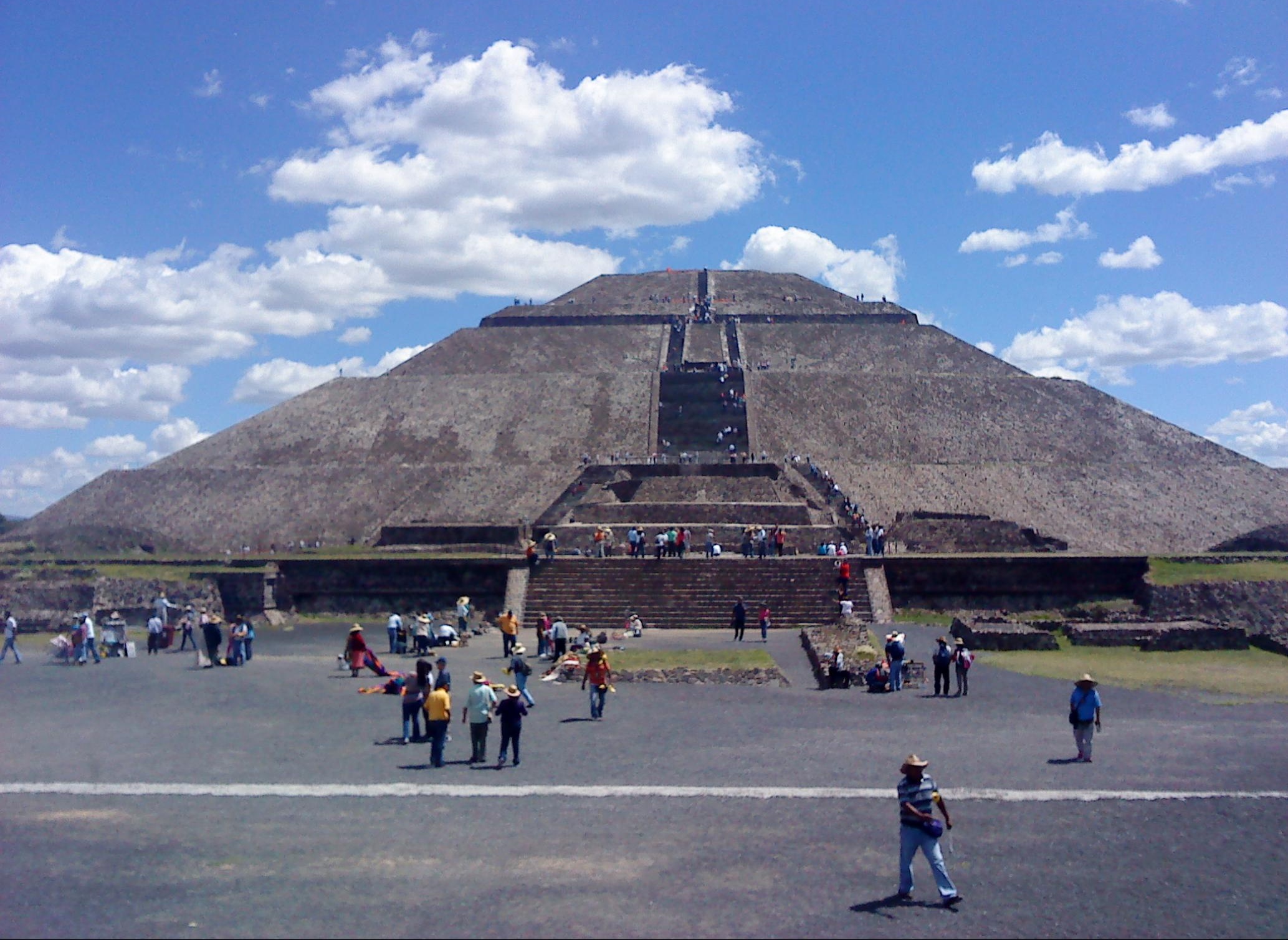

\title{
Sistemas de predicción por conjuntos en México
}

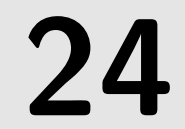

DOI: $10.31978 / 014-18-009-X .24$

\section{Servicio Meteorológico Nacional (SMN), México}

Nuestra misión: proveer pronósticos, alertas e información del estado del tiempo y del clima estratégica y útil para el país, que sustente la toma de decisiones. Nuestra visión: Seremos reconocidos por la sociedad como una organización efectiva y moderna que proporciona información confiable, útil y oportuna sobre meteorología y climatología para contribuir a una adecuada prevención y toma de decisiones, aplicando innovaciones tecnológicas y avances científicos con personal altamente calificado.

http://smn. cna.gob. mx/es/smn/mision-y-vision-SMN Presentamos en este capítulo el Sistema de predicción por conjuntos (SPC) que se está desarrollando en el Servicio Meteorológico Nacional de México, gracias a una moderna plataforma de servidores virtuales que describimos también con cierto detalle. Al igual que en el capítulo dedicado a Argentina, por el enorme interés cultural de la diversidad lingüística, se ha respetado la terminología original del trabajo, de uso al otro lado del Atlántico, aclarando algún término por facilitar la consistencia con otros capítulos de este volumen. El modelo atmosférico en que se basa este SPC es el mundialmente famoso Weather Research and Forecasting Model (WRF).

Palabras clave: sistemas de predicción por conjuntos en México, ensambles en servidor virtual.

Imagen parte superior: Tenochtitlán, octubre de 2012. Fotografía de CARLos SANTOS BURGUETE. 


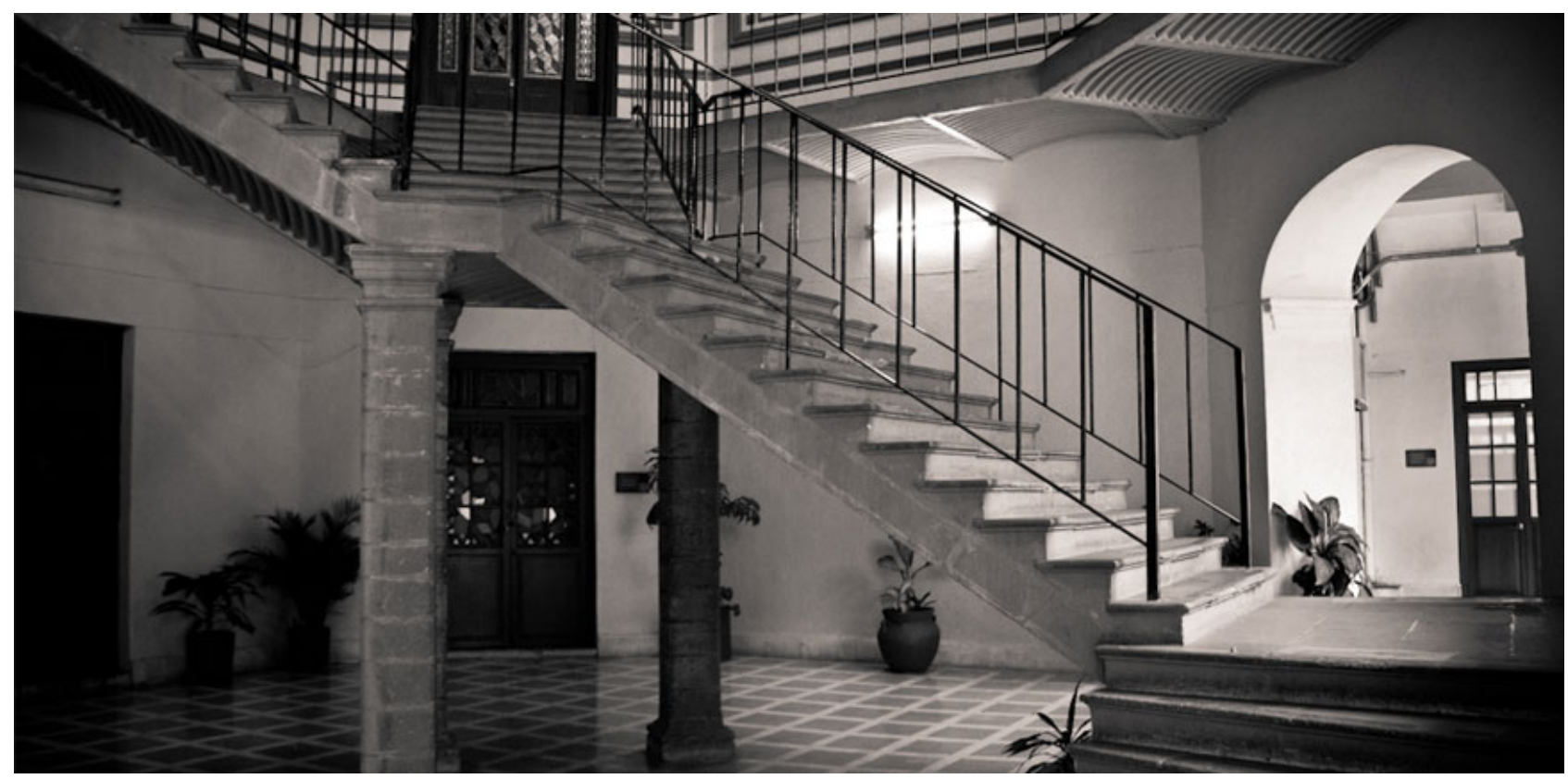

Figura 24.1: Servicio Meteorológico Nacional (SMN), escalera principal. Fotografía: http: //smn. cna. gob. $\mathrm{mx} / \mathrm{es} / \mathrm{smn} / \mathrm{historia}$

\subsection{Introducción}

El Servicio Meteorológico Nacional (SMN, Figuras 24.1 y 24.6 en la página 371) en México se creó el 6 de marzo de 1877 con la finalidad de dar un servicio integral a la inmigración extranjera, investigadores del campo, realizar un estudio exhaustivo de la Climatología del país, sus cambios atmosféricos, así como la de promover el intercambio internacional con otros observatorios y poner a disposición los datos recaudados a las personas y empresas que estén en aptitud de utilizarlos. Estas tareas aún se siguen manteniendo pero se han agregado más usuarios en las últimas décadas, como la industria turística, deportistas, instancias gubernamentales, empresas aseguradoras, etc. No hay en el mundo actualmente, algo que no tenga que ver con la meteorología y la climatología, de ahí la importancia de ser estudiada.

\subsection{Antecedentes}

El Servicio Meteorológico Nacional (SMN) preocupado por tener siempre el equipo idóneo para la medición, estudio, análisis y pronóstico de los procesos atmosféricos, a finales de la década de los 80 adquirió maquinas computadoras como la CYBER 380 y CYBER 920 donde se corría el Modelo Baroclínico de Tres Niveles elaborado por el Dr. Julian Adem Chahin [6] (http: //cronistadetuxpan.blogspot.mx/2011/05/ auditorium-del-centro-de-estudios-de-la. html). Tiempo después y dentro de un programa de modernización el SMN adquiere en el año 2000 una Supercomputadora Origin 2000 de 8 procesadores Reduced Instruction Set Computer (RISC) 12000 de Silicon Graphics, en donde se corrió durante 9 años el modelo Modelo a Mesoescala Generación 5 (MM5 [1]). En este lapso de tiempo se hicieron aportaciones a nivel Nacional e Internacional importantes como el cambio del uso de suelo para la Capital de la República Mexicana y presentar un caso de estudio [2] en el User's Workshop MM5 en el año 2002. Dado el costo de mantenimiento de este tipo de computadoras, en el año 2010 se adquieren una serie de CPU de la marca IBM que se instalan en la modalidad de Clúster, llegando a tener 25 procesadores, donde además de correr el modelo MM5 se incursionó en la siguiente generación de modelos a mesoescala, como el Weather Research and Forecasting Model (WRF [5]), además se graficaban los datos de entrada que corresponden al modelo Global Forecast System (GFS $[3,4])$. Teniendo la experiencia que las computadoras tiene un tiempo limitado de vida útil y sabiendo que se necesita un mejor pronóstico numérico del tiempo, surge la necesidad de tener corridas del modelo WRF por Ensamble es así que en el año 2013 bajo un convenio de colaboración con el Instituto Mexicano de Tecnología del Agua (IMTA), se implementa lo que se le conoce como: Corrida por Ensambles. 


\subsection{Primeros intentos}

Bajo esos términos se realizó un experimento que duró todo el año 2013, con un ensamble de 6 elementos (Figura 24.2), para una malla madre de resolución de $30 \mathrm{~km}$, que cubre todo el territorio Nacional. La aplicación se instaló en una maquina marca DELL con una configuración tipo Clúster que consta de un nodo maestro, 6 procesadores Intel Xeon con doble núcleo físico y doble núcleo virtual a una velocidad de $2.79 \mathrm{GHz}$ y memoria RAM de $24 \mathrm{~Gb}$, se tiene además
10 nodos clientes con las mismas características de procesador con la diferencia de una memoria RAM de 12 GB. Dicho equipo interconectado con red Ethernet y cuenta con un sistema de almacenamiento masivo de $65 \mathrm{~Tb}$ conectado al equipo mediante $10 \mathrm{Gbps}$, bajo el sistema operativo Centos. Esta computadora está por acabar su vida útil, lo que ahora se está haciendo es elaborar corridas operativas, aunque si publicar, por estar en periodo de prueba en Servidores Virtuales. Aquí se proyectó realizar un ensamble de 3 elementos, con equipo y en las instalaciones del SMN.

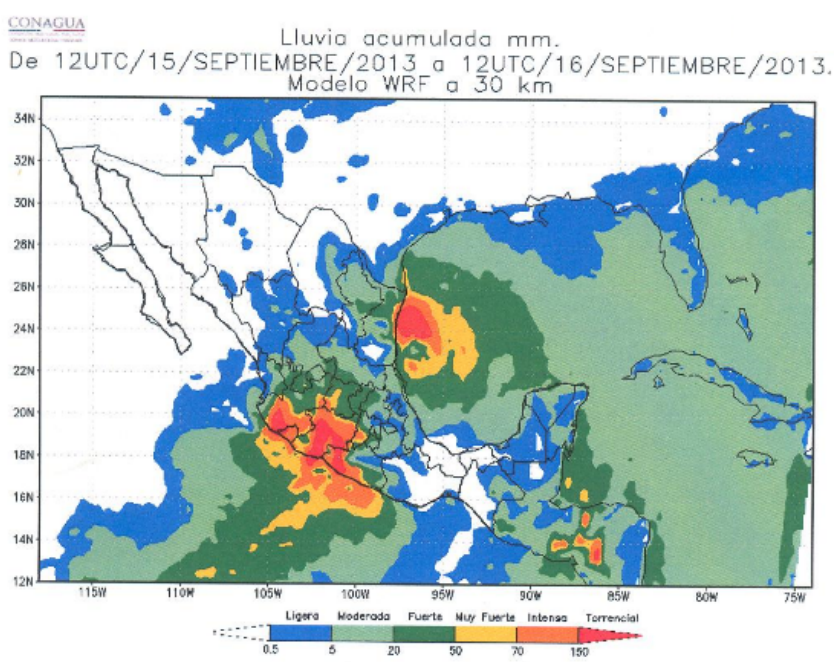

Conagua Promedio de Lluvia acumulada $\mathrm{mm}$

De 12UTC/15/SEPTIEMBRE/2013 a 12UTC/16/SEPTIEMBRE/2013. De 12UTC/15/SEPTIEMBRE/2013 a 12 UTC/16/SEPTIEMBRE/2013.
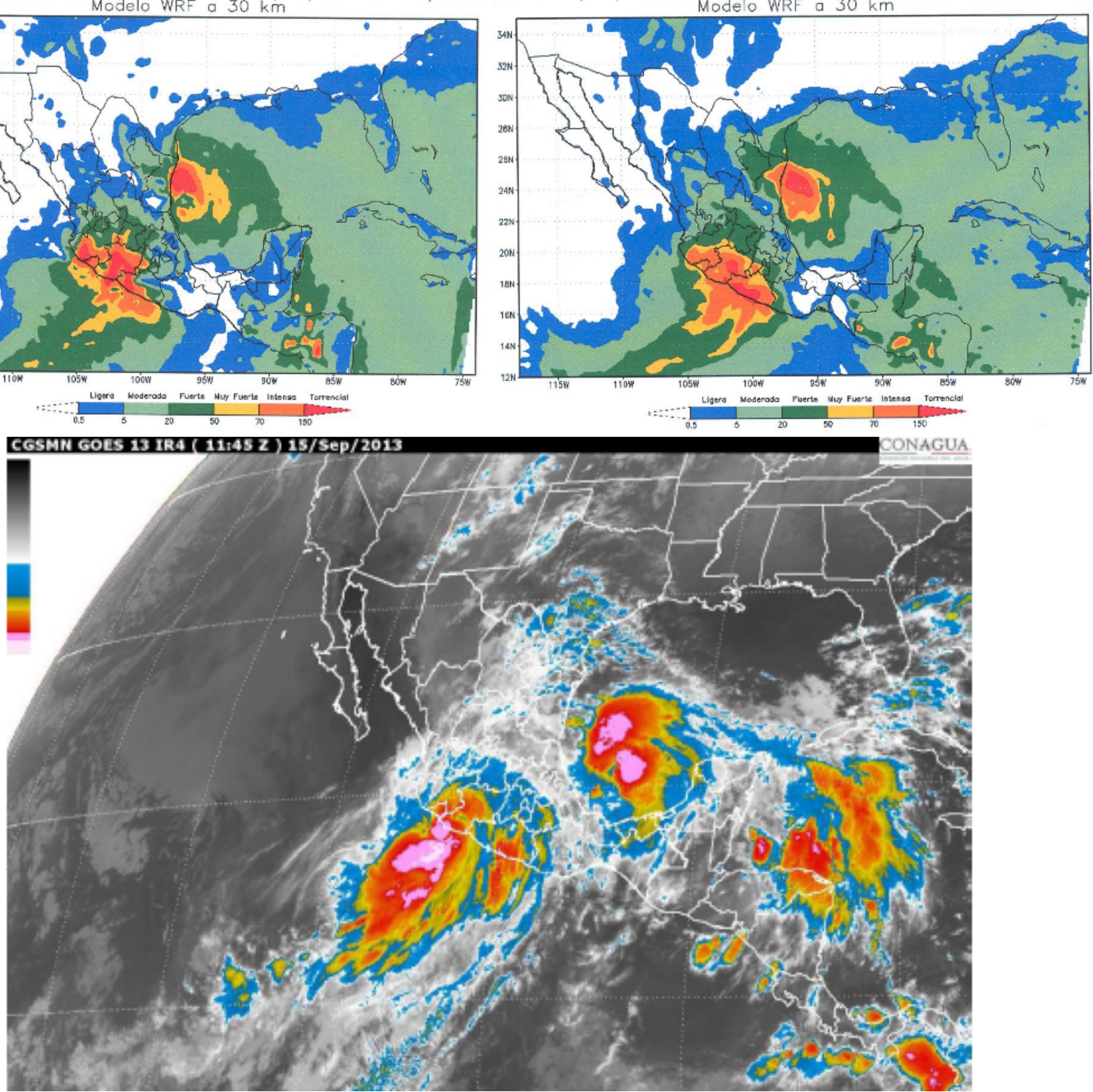

Figura 24.2: En la figura de la izquierda se observan los resultados del modelo WRF para una malla de $30 \mathrm{~km}$ con la parametrización KAIN-FRITSCH. En la carta de la derecha vemos el resultado del ensamble de 6 elementos. Abajo, la comparación real con imagen de Satélite de Canal Infrarrojo para el evento de los Huracanes MANUEL $e$ INGRID (GOES, CONAGUA). 


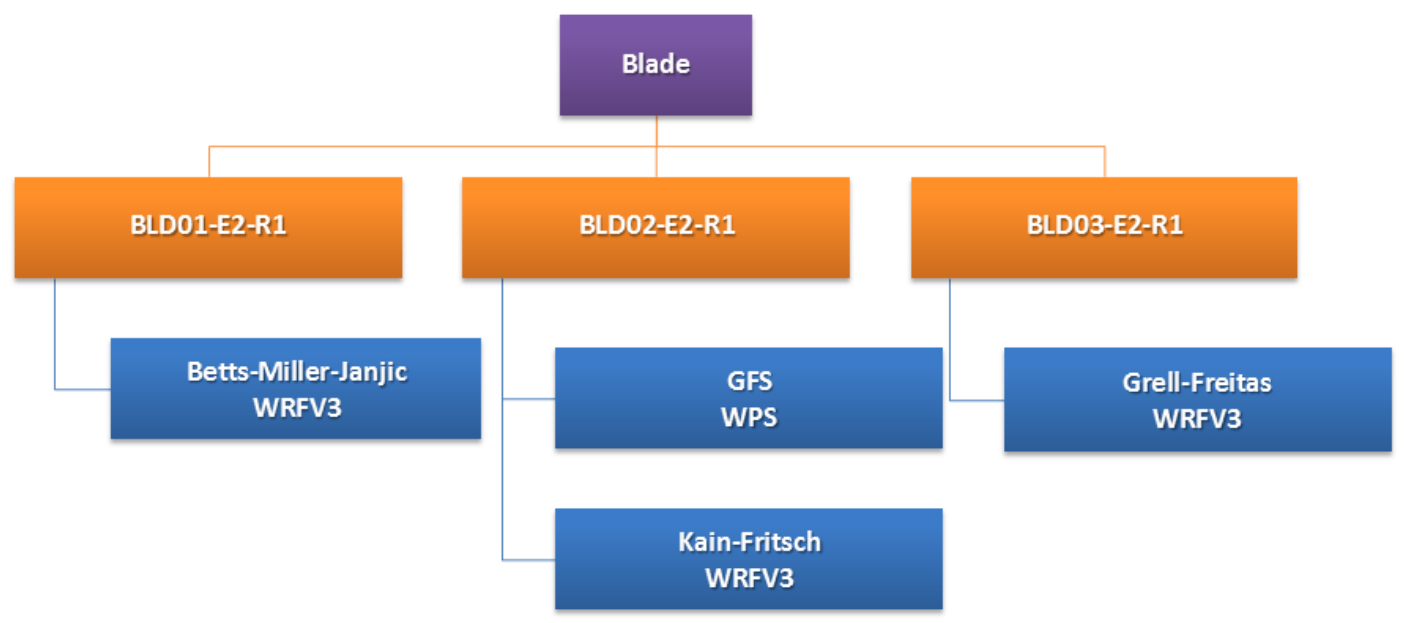

Figura 24.3: Arquitectura de distribución de máquinas virtuales en el SMN.

\subsection{Ensambles en Servidor Vir- tual}

Un Servidor Virtual Privado (VPS, por sus siglas en Inglés) es un método de particionar un servidor físico en varios servidores de tal forma que todo funcione como si se estuviese ejecutando en una única máquina. La implantación de los Servidores Virtuales en centros como los Servicios Meteorológicos ha tenido mucho auge, debido a una reducción de costos en la adquisición de equipo y su mantenimiento, administración más sencilla, mejor seguridad, flexibilidad, adaptable a medianas y grandes instituciones, un ahorro energético y de personal. Actualmente se trabaja con la configuración en las maquinas virtuales mostrada en la Tabla 24.1.

\begin{tabular}{|c|c|}
\hline Sistema operativo & CentOS 6.6 \\
\hline Memoria & 32 GB dinámicos \\
\hline Procesadores & 12 cores \\
\hline Discos Duros & 1 \\
\hline Disco Duro Virtual & $\begin{array}{c}\text { 300 GB para el Sistema } \\
\text { Operativo }\end{array}$ \\
\hline Conexiones & VNC y SSH \\
\hline
\end{tabular}

Tabla 24.1: Configuración en las máquinas virtuales.

Se tiene instalado y configurado el modelo WRF, versión 3.6.1, para el desarrollo de un pronóstico a $72 \mathrm{~h}$ con salidas cada $3 \mathrm{~h}$ con una malla de $16 \mathrm{~km}$ de resolución, los datos de entrada son del modelo GFS de $0.5^{\circ}$ (aproximadamente $25 \mathrm{~km}$ de resolución), las parametrizaciones por el momento son: KAIN-FRITSCH, BETTS-MILLER-JANJIC y GRELL-FreITAS, para los tiempos de las salidas del modelo GFS de 00 UTC, 06 UTC, 12 UTC y 18 UTC. La arquitectura de distribución de dichas máquinas virtuales puede verse en la Figura 24.3.

Esta implementación nos ha estado arrojando muy buenos resultados en cuanto a los tiempos de procesamiento comparándose con el tiempo de procesamiento del modelo WRF instalado en un clúster (ver capítulo 11 en la página 145). En la Figura 24.4 se muestran unas tablas con los tiempos obtenidos de las pruebas realizadas, cabe aclarar que las horas de inicio y fin están dadas en horario local.

En la Figura 24.5 en la página siguiente se exhiben algunos ejemplos de los productos que se generan actualmente en el SMN, en el modo de Servidores Virtuales.

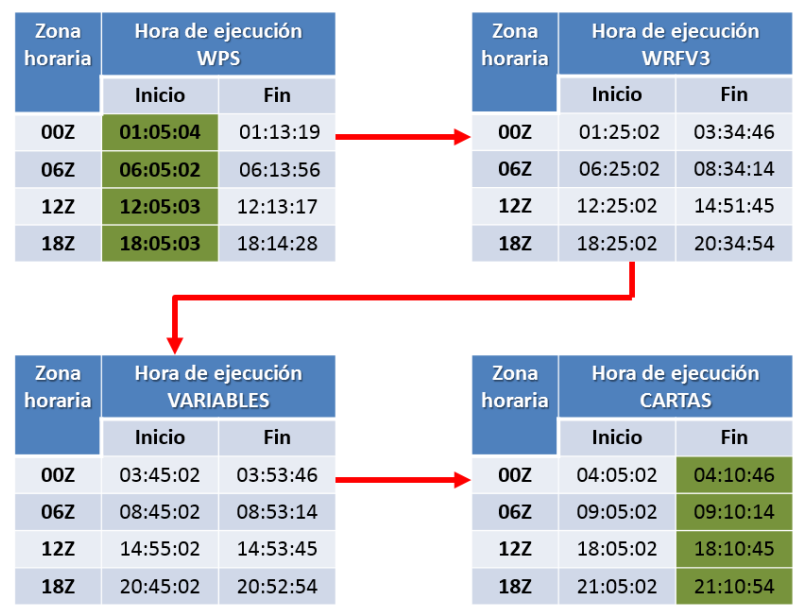

Figura 24.4: Rendimiento y tiempos obtenidos con las pruebas en las máquinas virtuales en el SMN. 

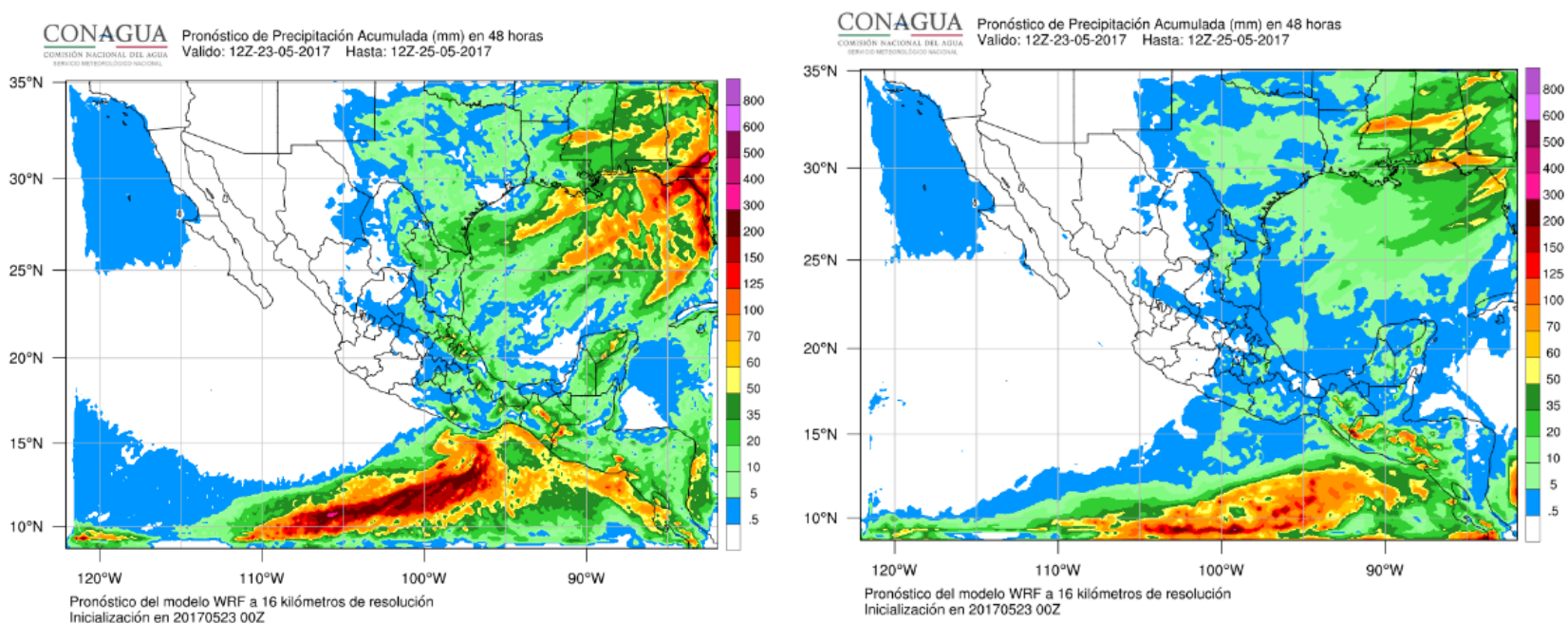

Figura 24.5: La imagen de la izquierda es el resultado del modelo WRF con la parametrización KAIN-FRITSCH. A la derecha con la parametrización BETTS-MILLER-JANJIC.

\subsection{Futuro}

El pronóstico numérico actualmente es una de las herramientas más poderosa e imprescindible en los Servicios Meteorológicos Nacionales, sin embargo en modelación todavía se trata de alcanzar un buen porcentaje de acierto en la previsión atmosférica. Los esfuerzos han sido muchos, se ha avanzado demasiado pero falta mucho por hacer. El SMN en un futuro próximo contara con el pronóstico por Ensambles con el fin de seguir colaborando con el pronosticador para que éste realice mejores análisis, pronósticos aún más eficaces, confiables y en un tiempo relativamente corto, beneficiando con esto a los usuarios internos y externos al Servicio Meteorológico Nacional y al pueblo de México en general, contribuyendo a tomar las medidas precautorias en eventos atmosféricos severos, como se nos indicó desde hace más de 140 años.

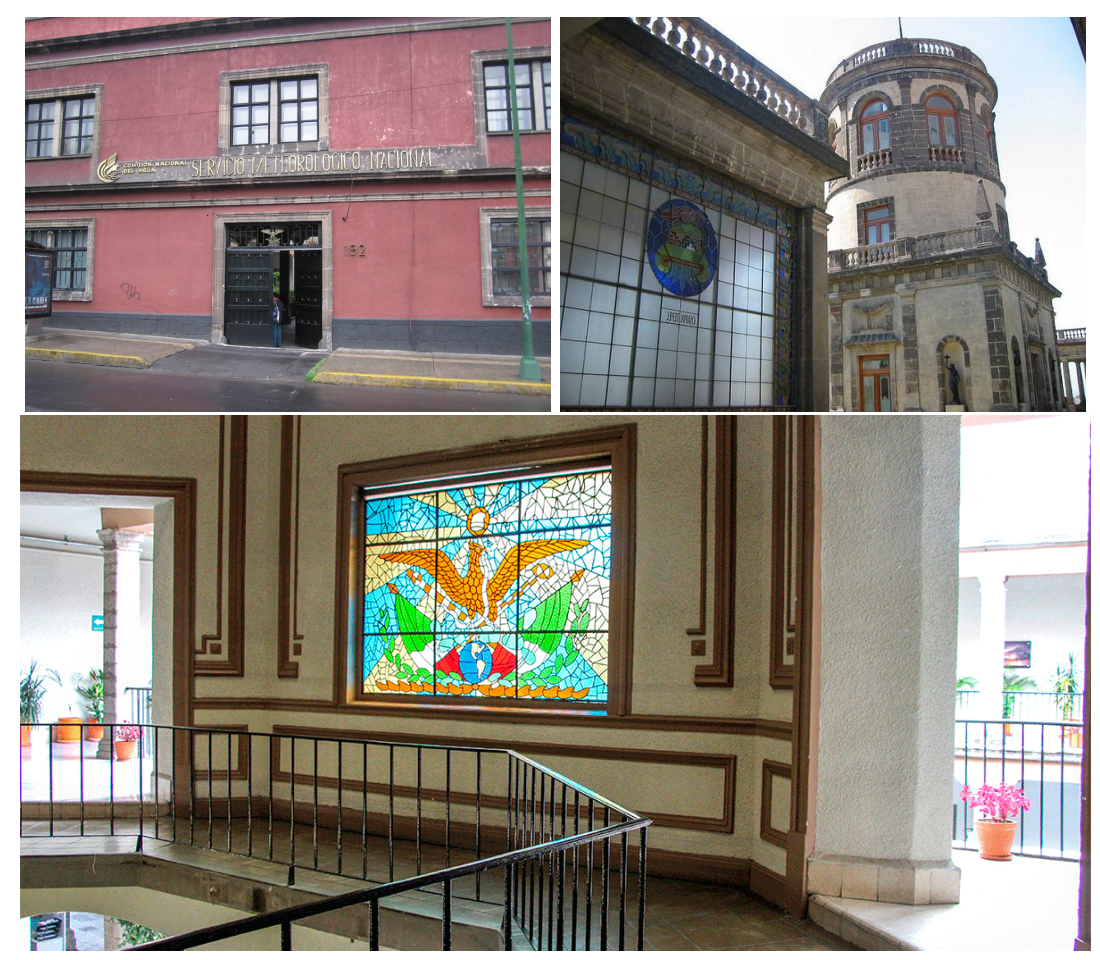

Figura 24.6: Imágenes del Servicio Meteorológico Nacional. 


\subsection{Referencias}

[1] Grell, Georg A, Dudhia, Jimy, StaufFER, David R y col. "A description of the fifth-generation Penn State/NCAR mesoscale model (MM5)". En: (1994) (citado en página 368).

[2] LobAto, René y col. "MM5 model validation under different cumulus parameterizations". En: Proceedings of the 12th. PSU/NCAR MM5 User's Workshop. 2002 (citado en página 368).

[3] Sela, Joseph G. "Spectral modeling at the national meteorological center". En: Monthly Weather Review 108.9 (1980), páginas 1279-1292 (citado en página 368).
[4] SELA, Joseph G. "The NMC spectral model". En: NOAA Technical Report, NWS-30 108 (1982) (citado en página 368).

[5] Skamarock, William C y col. A description of the advanced research WRF version 2. Informe técnico. National Center For Atmospheric Research Boulder Co Mesoscale y Microscale Meteorology Div, 2005 (citado en página 368 ).

[6] Zamora SÁNCHeZ, Obed. Tuxpan, las crónicas de su ciudad y puerto: homenaje a Julián Adem Chahin en la UNAM. URL: http: / / cronistadetuxpan . blogspot . com . es/2011/05/auditorium-del-centrode - estudios - de - la . html (visitado 01-02-2018) (citado en página 368). 
